\title{
sensors
}

ISSN 1424-8220

(C) 2002 by MDPI

http://www.mdpi.net/sensors

\section{A First Step Towards a Microfabricated Thin-Film Sensor Array on the Basis of Chalcogenide Glass Materials}

\author{
Joachim P. Kloock ${ }^{1,2}$, Yulia G. Mourzina ${ }^{1,3}$, Jürgen Schubert ${ }^{1}$ and Michael J. Schöning ${ }^{1,2, *}$ \\ ${ }^{1}$ Department of Applied Physics, University of Applied Sciences Aachen, Ginsterweg 1, 52428 Jülich, \\ Germany \\ ${ }^{2}$ Institute for Thin Films and Interfaces, Research Centre Jülich GmbH, 52425 Jülich, Germany \\ ${ }^{3}$ Department of Chemistry, St. Petersburg State University, Universitetskaya nab. 7/9, St. Petersburg \\ 199034, Russia \\ * Author to whom correspondence should be addressed. Tel: +49/2461/612973. Fax:+49/2461/612940. \\ E-mail: M.J.Schoening@fz-juelich.de
}

Received: 19 August 2002 /Accepted: 1 September 2002 / Published: 16 September 2002

\begin{abstract}
A first step towards a microfabricated potentiometric thin-film sensor array for the simultaneous detection of $\mathrm{Pb}^{2+}, \mathrm{Cd}^{2+}$ and $\mathrm{Cu}^{2+}$ has been realized. The sensitive layers used are on the basis of chalcogenide glass materials. These thin-film chalcogenide glass materials that consist of mixtures of Pb-Ag-As-I-S, Cd-Ag-As-I-S or Cu-Ag-As-Se have been prepared by pulsed laser deposition technique. The developed sensor array has been physically characterized by means of scanning electron microscopy and Rutherford backscattering spectrometry. The electrochemical sensor characterization has been performend by potentiometric measurements.
\end{abstract}

Keywords: heavy metal detection, thin-film sensor array, chalcogenide glass material, microfabrication technique, pulsed laser deposition, potentiometry.

\section{Introduction}

Nowadays, the development of cost-effective sensors and sensor systems for analytical tasks becomes more and more important. Under the aspect of a growing demand for environmental analysis as well as monitoring and process control systems, the request of these market segments are an easy to 
use and cheap realization of sensor systems. The trend of such sensor systems goes into the direction of multisensor arrangements, so-called sensor arrays [1-3].

In the field of analytics of aqueous solutions, recently, the term of "electronic tongue" was created. In common, an "electronic tongue" sensor system consists of the sensor- and the signal/dataprocessing unit. The sensor part is often an array of non-specific, poorly selective sensors with crosssensitivities to different elements in the test samples. In combination with pattern recognition methods, like fuzzy logic or artificial neural networks, the electronic tongue can become a powerful analytical instrument [4-6]. One attempt for the fabrication of such "electronic tongue"-based sensor systems for the detection of metal ions $\left(\mathrm{Pb}^{2+}, \mathrm{Cd}^{2+}, \mathrm{Cu}^{2+}, \mathrm{Tl}^{+}\right.$, etc.) in aqueous media on the basis of the ionselective potentiometry offer chalcogenide glasses. The advantages of chalcogenide glasses are their good stability in corrosive media and their long-term stability, to only give two examples [7]. Conventional bulk electrodes on the basis of chalcogenide glass materials are available from Analytical Systems, St. Petersburg. Investigations of a macroscopic sensor array based on these conventional chalcogenide glass bulk electrodes already exist and the results are published in [8]. However, the disadvantage of this sensor system is the expensive fabrication of the single electrodes as well as the insufficient possibility of miniaturization of the sensor array by means of batch processes.

To solve these problems in terms of realizing a compact and cheap sensor system, it is necessary to deposit these chalcogenide glass materials as thin films in a modular set-up. As a new tool to deposit such complex materials, the pulsed laser deposition (PLD) technique has been recently suggested. The major advantage of the PLD is the stoichiometric transfer of the complex target materials such as chalcogenide glasses ( $\mathrm{Pb}$-Ag-As-I-S, Cd-Ag-As-I-S, etc.) to a given substrate, the short process times and the fabrication compatibility to methods of silicon planar technology $[9,10]$.

By means of the application of chalcogenide glass materials as ion-selective layers together with the combination of the PLD process and silicon planar technology for material processing and structuring, we can demonstrate that it is possible to design a compact sensor array for the detection of $\mathrm{Pb}^{2+}-\mathrm{Cd}^{2+}$ and $\mathrm{Cu}^{2+}$-ions in aqueous solutions onto one silicon substrate. This sensor array represents a first step towards a microbabricated thin-film sensor array on the basis of chalcogenide glass materials into the direction of an "electronic tongue"-based sensor system.

\section{Experimental}

\section{Fabrication of the Chalcogenide Glass-Based Thin-Film Sensor Array}

The chalcogenide glasses, which are needed as targets for the subsequent PLD process, were synthesized from the base elements of high purity in evacuated quartz ampoules at a pressure between 0.1-0.01 $\mathrm{Pa}$ and at temperatures of 1000-1200 K for 5-12 $\mathrm{h}$. The ampoules with the melt were airquenched in the 400-600 $\mathrm{K}$ temperature range, with an average cooling rate of 4-6 $\mathrm{Ks}^{-1}$ [7,11]. In this way prepared chalcogenide glasses served as target materials for the thin-film deposition.

For the fabrication of the sensor arrays, a p-doped (Bor) 4" single-crystal silicon wafer (WackerChemitronic) with $<100>$-orientation, specific resistance of $>1000 \Omega \mathrm{cm}$ and thickness of $381 \pm 25 \mu \mathrm{m}$ 
has been used. The complete fabrication steps to realize the thin-film sensor array are summarized in Figure 1.

(I) Generation of $\mathrm{SiO}_{2}$
by thermal oxidation

Photoresist by spin-coating

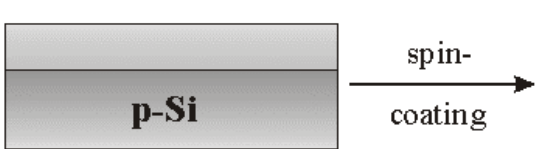

(VI) Lift-off process

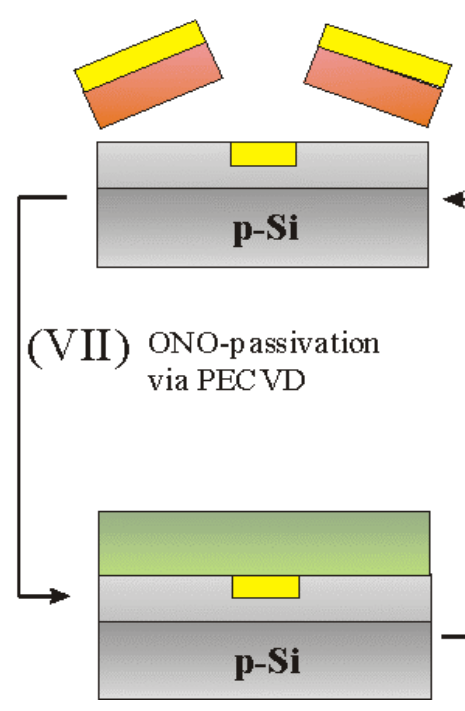

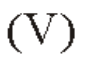

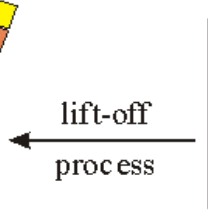

(VIII)

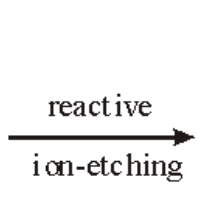

Contact layer Ti:Pt:Au

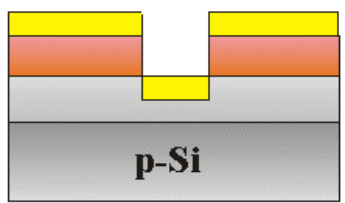

Open the electrodes by RIE
(III)
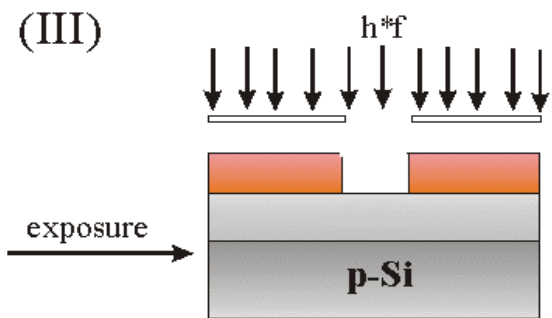

(IV)

Chemical etching

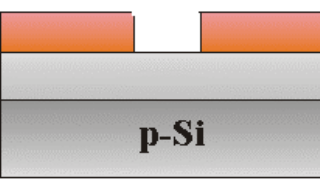

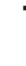

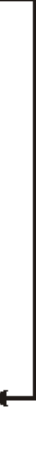

(IX)

Chal cogen ide glass via pulsed laser dep osition
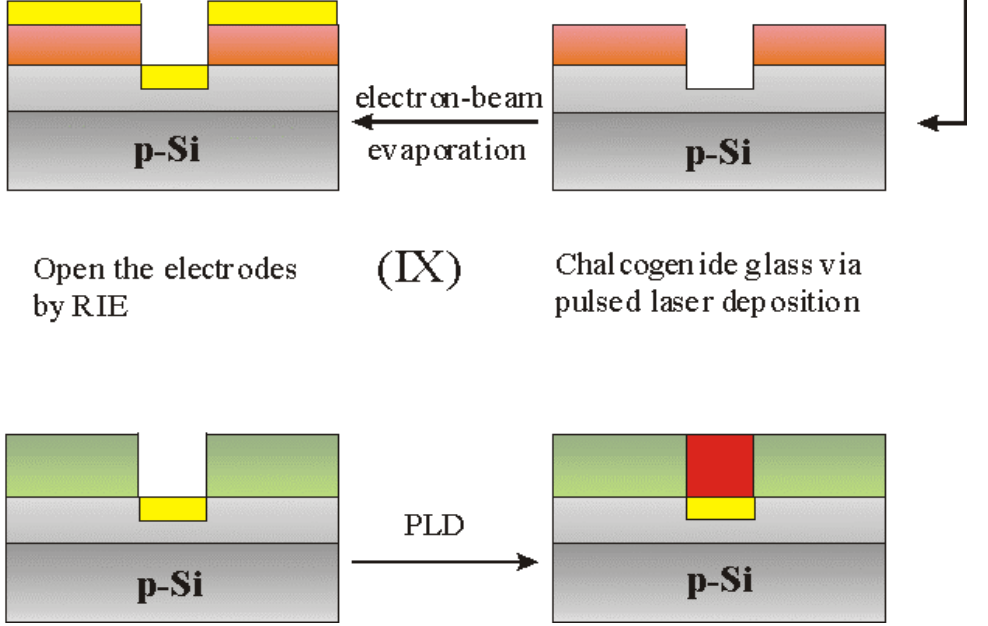

Figure 1. Survey on the fabrication steps of the chalcogenide glass-based thin-film sensor array (I: thermal oxidation, II: spin-coating, III: exposure, IV: chemical etching, V: electronbeam evaporation, VI: lift-off process, VII: ONO passivation, VIII: RIE, IX: PLD).

The first fabrication step is the generation of a $\mathrm{SiO}_{2}$ layer with a thickness of about $500 \mathrm{~nm}$ as insulating layer between the substrate and the electrodes as well as the electrodes (fabrication step I). This $\mathrm{SiO}_{2}$ layer was grown by means of thermal oxidation (oxidation oven, Tempress). To structurize the respective thin-film sensors as sensor array on the silicon wafer, photolithographic patterning has been performend (fabrication steps II + III). Therefore, a positive photoresist (AZ 5214, Hoechst) was spin-coated on the silicon substrate. Hexamethyldisiloxan was firstly used to improve the adhesion between the photoresist and the silicon substrate. For the exposure of the photoresist through a mask, which consists of the structure of the sensor array, a mask aligner (MA 6, Suss-microtec) was used. After developing the photoresist (AZ 312 MIF developer, Clariant), the structure of the sensor electrodes was chemically wet-etched by hydrofluoric solution (AF-91-09-HF, Riedel de Haen) as shown in fabrication step IV. In this way, trenches have been etched into the $\mathrm{SiO}_{2}$ layer, in order to embed the contact layers for the sensor structures. Fabrication step V depicts the deposition of the contact layers by means of electron-beam evaporation (PLS 500, Balzers). The contact layers are builtup of a layer system of Ti:Pt:Au with a thickness of 30:175:300 nm. 
The deposition of the contact layers has been finished by a lift-off process in acetone that allows to remove all photoresist-coated metallic areas (see fabrication step VI). To prevent a later corrosion of the silicon substrate and the contact layers, an oxide-nitride-oxide (ONO) passivation layer system has been deposited by means of a plasma-enhanced chemical vapour deposition process (UDP 80, Plasma Technology) [12]. This ONO-layer system was made of $\mathrm{SiO}_{2}: \mathrm{Si}_{3} \mathrm{~N}_{4}: \mathrm{SiO}_{2}$ with a thickness of 130:530:130 nm (see fabrication step VII). By means of a reactive ion-etching process (AMR, Oxford Instruments), only the areas which serve as contact for the sensor layers as well as the contact pads have been opened, as shown in fabrication step VIII.

Subsequent to the described process steps, the heavy metal-selective chalcogenide glass materials such as Pb-Ag-As-I-S, Cd-Ag-As-I-S and Cu-Ag-As-Se have been deposited by means of an "offaxis" PLD-process (see fabrication step IX). A KrF-excimer laser (LPX 300, Lambda) with a wavelenght of $248 \mathrm{~nm}$ and a repetition rate of $10 \mathrm{~s}^{-1}$ was used to deposit the individual materials of the respective chalcogenide glass target to the $\mathrm{Si} / \mathrm{SiO}_{2}$ substrate including the contact layers. An energy density of the laser of $5 \mathrm{Jcm}^{-2}$ was applied to the chalcogenide glass target at room temperature. The PLD process took place in a $\mathrm{N}_{2}$-atmosphere to prevent any oxidation of the deposited materials at a pressure of $2 * 10^{-1}$ mbar. A process time of approximately 10 minutes was used to get thin films with a thickness of about $500 \mathrm{~nm}$. To ensure the patterning of the different thin-film sensors on the silicon chip, a home-made Al mask has been used. The detailed PLD process is described elsewhere $[9,10,13]$. After cutting the wafer into single sensor chips of $10 \mathrm{~mm} * 10 \mathrm{~mm}$, each sensor chip has been glued on a printed circuit board (PCB), wire-bonded and encapsulated by an epoxy resin (EPO-TEK 87-GT, Polytec). Figure 2 shows the cross-sectional view of a single sensor chip including the chalcogenide glass-based thin-film sensor array after connection to the PCB and encapsulation.

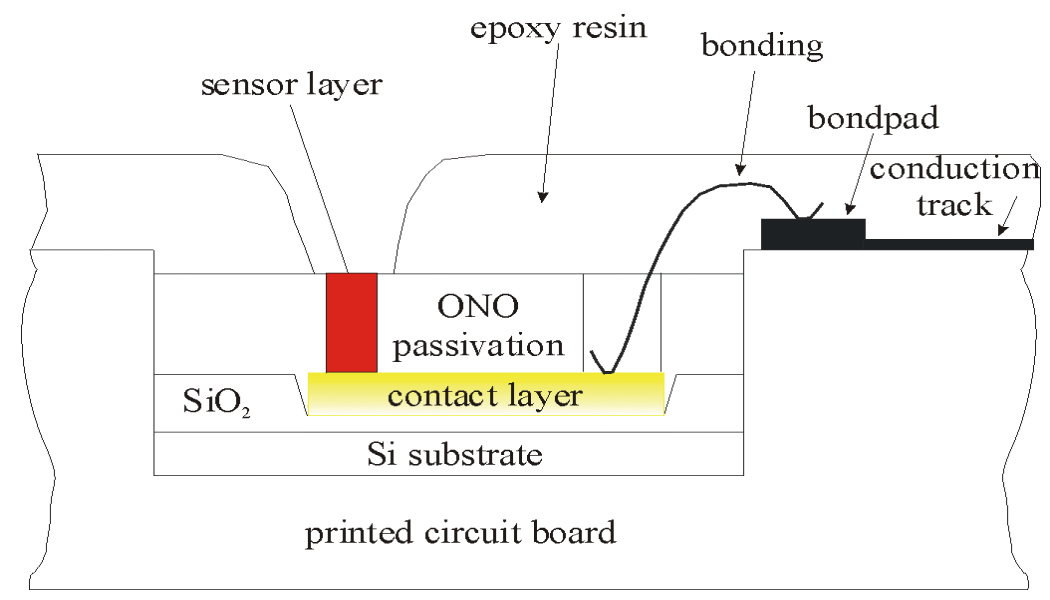

Figure 2. Schematic cross-section of the chalcogenide glass-based sensor chip after bonding and encapsulation.

Physical and Electrochemical Characterization of the Chalcogenide Glass-Based Thin-Film Sensor Array

The thin films based on chalcogenide glass materials were physically studied using Rutherford backscattering spectrometry (RBS) to control the stoichiometry of the target and the deposited thin 
films. By means of transmission electron microscopy (TEM) as well as scanning electron microscopy (SEM) the morphology and topography of the prepared thin films have been proved. For the TEM characterization, see e.g. [10].

The electrochemical properties of the sensor array were investigated by means of potentiometric measurements. The sensor array itself represents an electrochemical half-cell. To complete the measuring set-up for ion-selective potentiometry, it is necessary to utilize an additional reference electrode. In this experiment, we chose a $\mathrm{Ag} / \mathrm{AgCl}$ double-liquid junction reference electrode with an inner solution of $0.1 \mathrm{~mol} / \mathrm{l} \mathrm{KCl}$, and $0.1 \mathrm{~mol} / 1 \mathrm{KNO}_{3}$ for the outer solution. The potentiometric response of the sensor array has been characterized in $\mathrm{Pb}^{2+}-\mathrm{Cd}^{2+}$ and $\mathrm{Cu}^{2+}$-solutions in the concentration range of $10^{-6}-10^{-2} \mathrm{~mol} / 1$ for $\mathrm{Pb}^{2+}$ - and $\mathrm{Cd}^{2+}$-ions, respectively, and $10^{-7}-10^{-3} \mathrm{~mol} / 1$ for $\mathrm{Cu}^{2+}$-ions. To guarantee a constant ionic strength of the test sample, a constant background electrolyte solution of $0.1 \mathrm{~mol} / 1 \mathrm{KNO}_{3}$ was used. All chemicals applied were of reagent grade.

The sensor signal was recorded by a highly ohmic voltmeter (Type 2700, Keithley) controlled by a conventional personal computer via general purpose interface bus (GPIB); using a multiplexer offers the possibility to simultaneously measure and control up to 20 sensors. A schematic picture of the complete measurement set-up is shown in Figure 3.

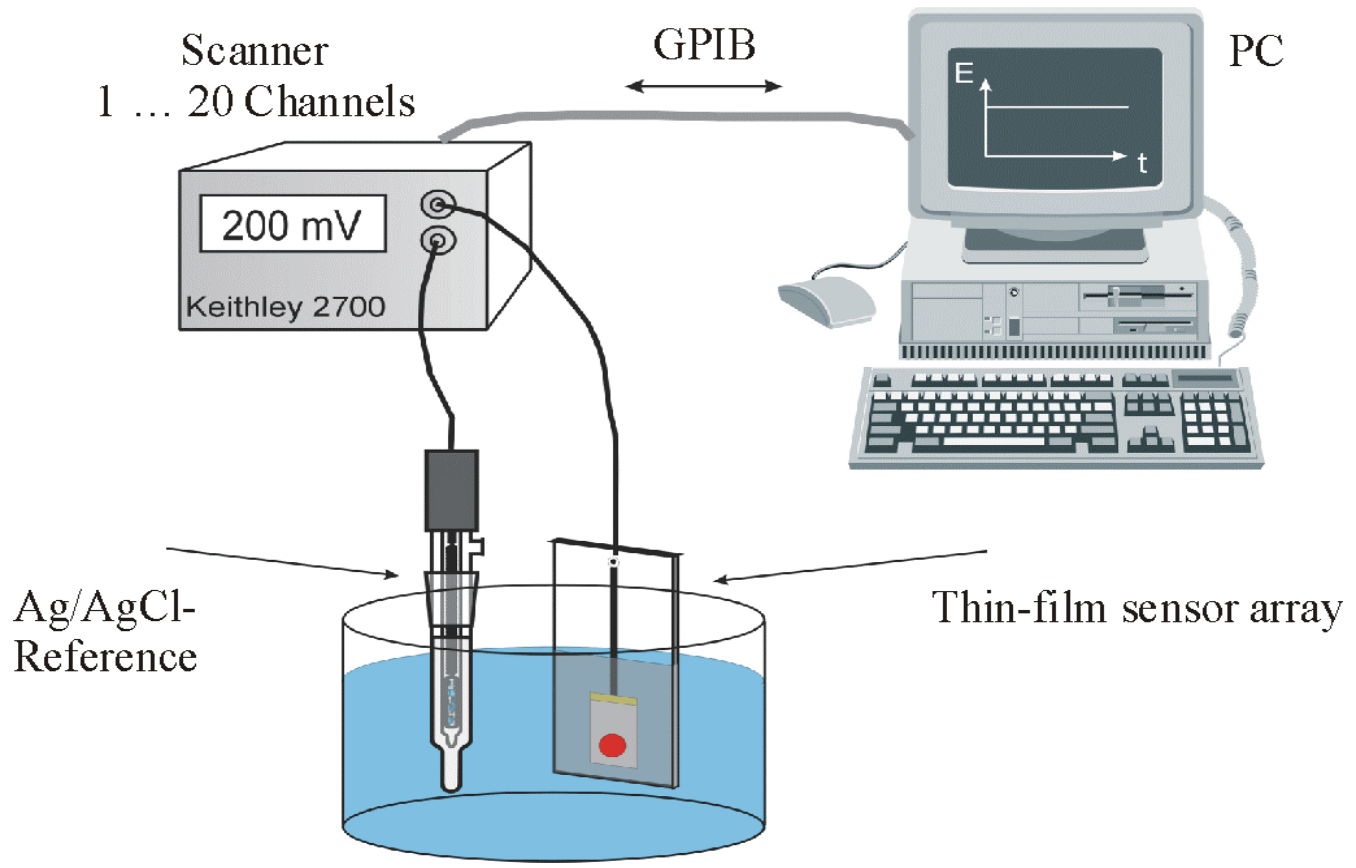

Figure 3. Measurement set-up for the electrochemical characterization of the chalcogenide glass-based thin-film sensor array.

\section{Results and Discussion}

Physical Characterization of the Chalcogenide Glass-Based Thin-Film Sensor Array

A videomicroscopic picture of the thin-film sensor array after the PLD process is given in Figure 4 (a). The $10 \mathrm{~mm} * 10 \mathrm{~mm}$ silicon chip carries the different chalcogenide glass thin films and a 
pseudo-reference. For the electrical connection to the PCB, the bond pads are shown in the lower part of the picture. The $\mathrm{Pb}$-selective sensor layer is located on the left electrode, the Cd-selective sensor layer on the right electrode and the $\mathrm{Cu}$-selective sensor layer on the lower middle electrode. In the middle of the sensor array, the pseudo-reference, which consists of a gold layer, is located. This pseudo-reference has not been used in this experiment for the potentiometric characterization of the thin-film sensor array. For the potentiometric measurements, a $\mathrm{Ag} / \mathrm{AgCl}$ double-liquid junction reference electrode has been utilized. The diameter of electrodes is about $2 \mathrm{~mm}$. The slight colour difference between the bond pads and the conduction tracks depends on the thickness of the ONOpassivation layer system.

In Figure 4 (b), the complete thin-film sensor array is presented after wire bonding and encapsulation. The sensor array from Figure 4 (a) was now sticked onto the PCB, bonded and encapsulated with epoxy resin. In the left part of the picture, the conduction tracks and the contact pins to the measurement device can be seen.

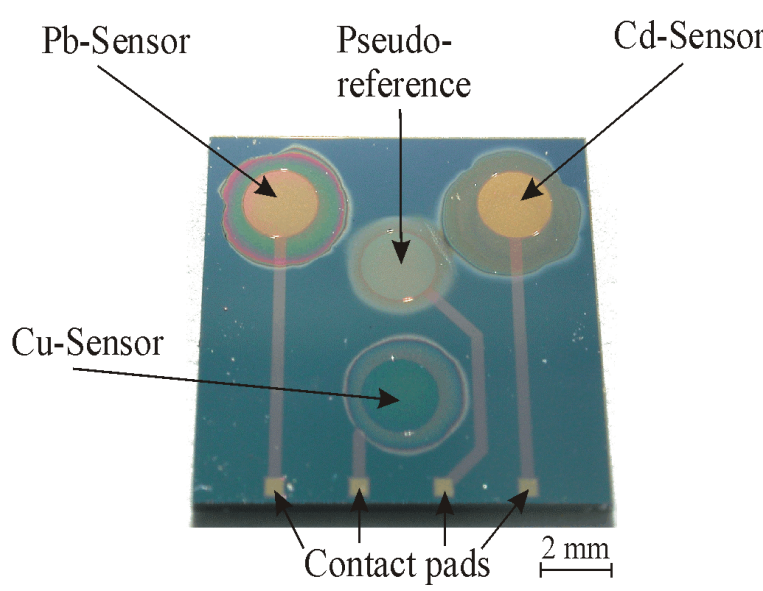

(a)

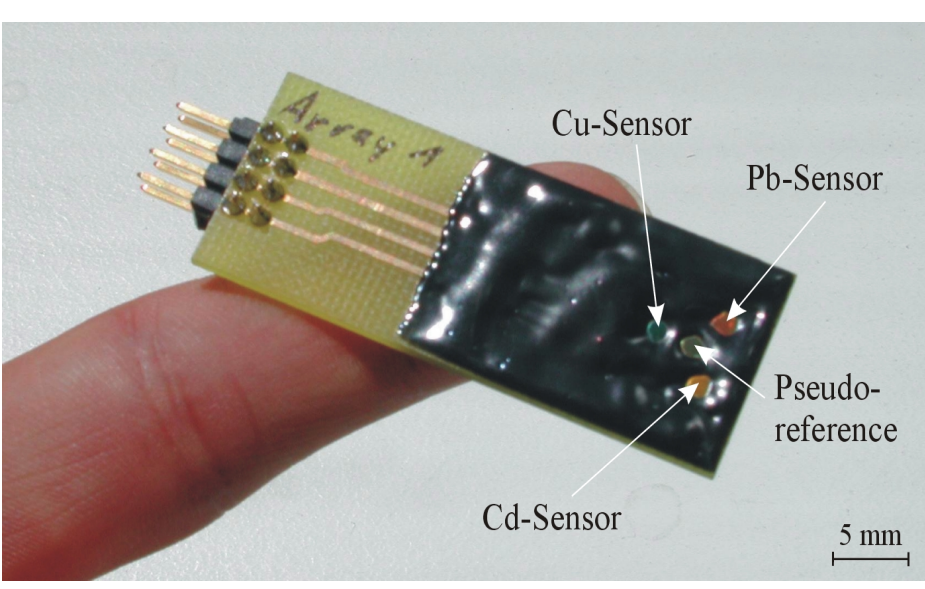

(b)

Figure 4. (a) Videomicroscopic picture of the thin-film sensor array after the PLD process. The different chalcogenide glasses onto the electrodes are shown. (b) Picture of the complete thin-film sensor array after wire bonding and encapsulation.

To study the morphology of the thin films, SEM investigations have been done. In Figure 5, a

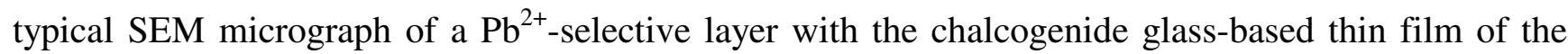
system $\mathrm{Pb}$-Ag-As-I-S is shown. The surface of the deposited thin-film material has a nearly homogeneous distribution of the chalcogenide glass on the electrode surface.

No distinct crystallographic orientation of the thin-film layer has been found. The stoichiometric deposition has been proven by means of RBS experiments (see also Figure 6). On the SEM micrograph, one can also recognize so-called droplets, i.e. clusters of the deposited target material that is typical for PLD-prepared thin-film layers. However, these droplets completely cover the sensor surface and do not influence both the stoichiometry and the electrochemical sensor performance of the respective sensor within the thin-film sensor array. For all other chalcogenide glass materials investigated in this experiment, the SEM micrographs are quite similar. 


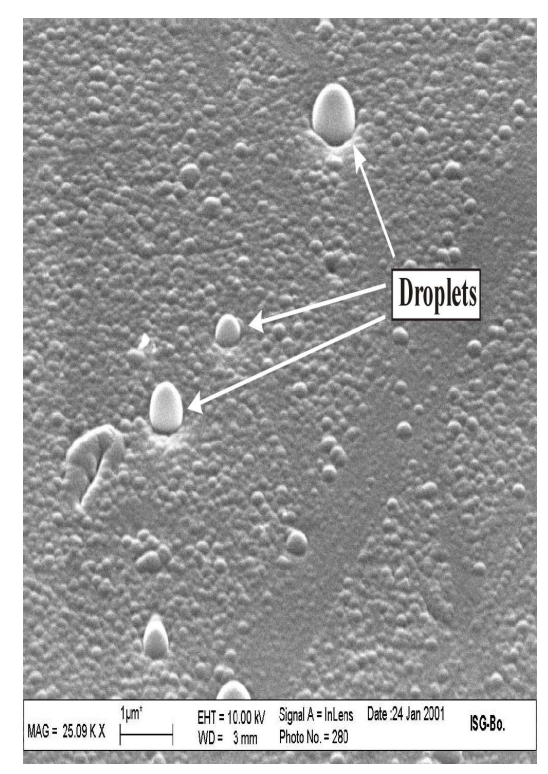

Figure 5. SEM micrograph of the surface of the $\mathrm{Pb}^{2+}$-selective layer of the thin-film sensor array with chalcogenide glass material system of $\mathrm{Pb}-\mathrm{Ag}$-As-I-S.

In Figure 6, a typical RBS measurement is exemplarily presented for the chalcogenide glass layer system of Pb-Ag-As-I-S onto a silicon substrate. The RBS measurements in this work could validate the stoichiometric transfer of the chalcogenide glass target to the substrate as thin film by means of the PLD process. In the figure, the elemental composition of the thin film was compared to the simulation based on the bulk material. As can be seen, there is a good correlation between the measurement data of the thin film and the simulation data. Thus, a nearly perfect stoichiometric transfer from the target material to the silicon substrate can be assumed. Former investigations in terms of the thin-film composition, also done by the authors, are published elsewhere $[9,10,13,14]$.

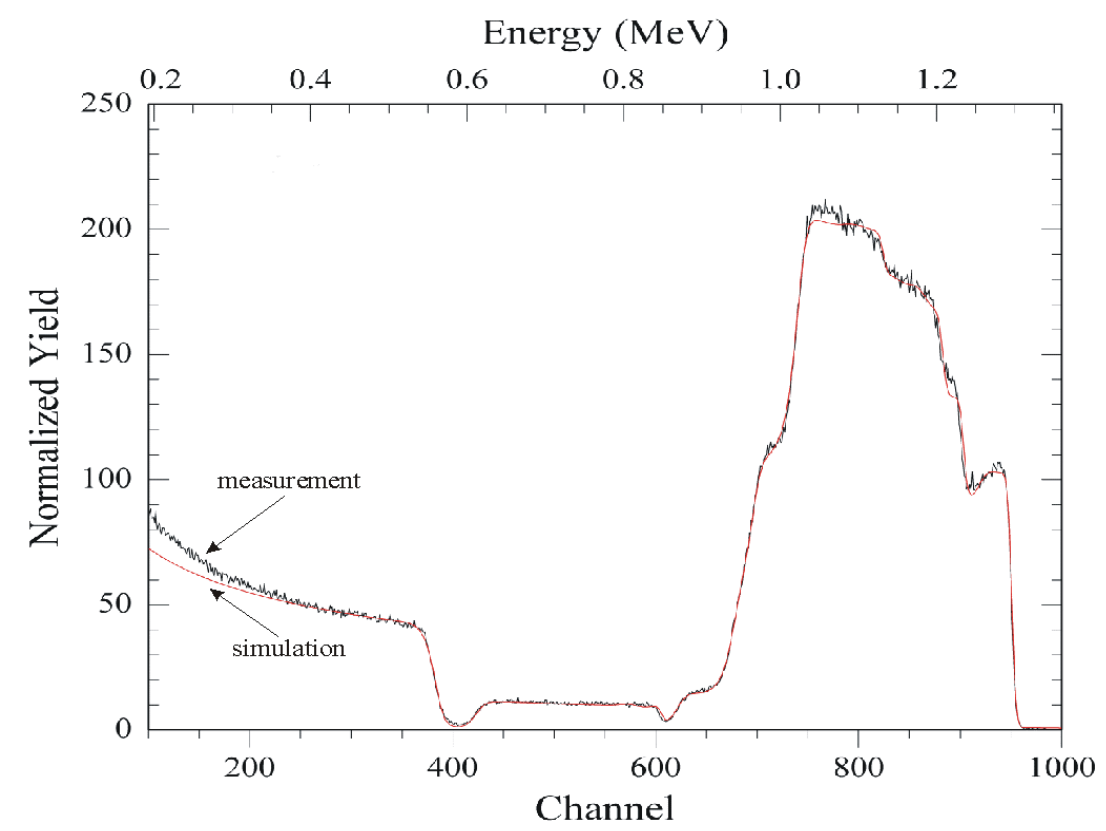

Figure 6: RBS measurement of chalcogenide glass layer system of $\mathrm{Pb}-\mathrm{Ag}$-As-I-S onto a silicon substrate. 


\section{Electrochemical Characterization of the Chalcogenide Glass-Based Thin-Film Sensor Array}

The chalcogenide glass-based sensor array was electrochemically characterized by means of potentiomentric measurements. Measurements with different ionic solutions (variable ion concentrations) with variable lead, cadmium and copper contents have been performed. The response of the sensor array for measurements in $\mathrm{Cd}\left(\mathrm{NO}_{3}\right)_{2}$-solutions is exemplarily shown in Figure 7.

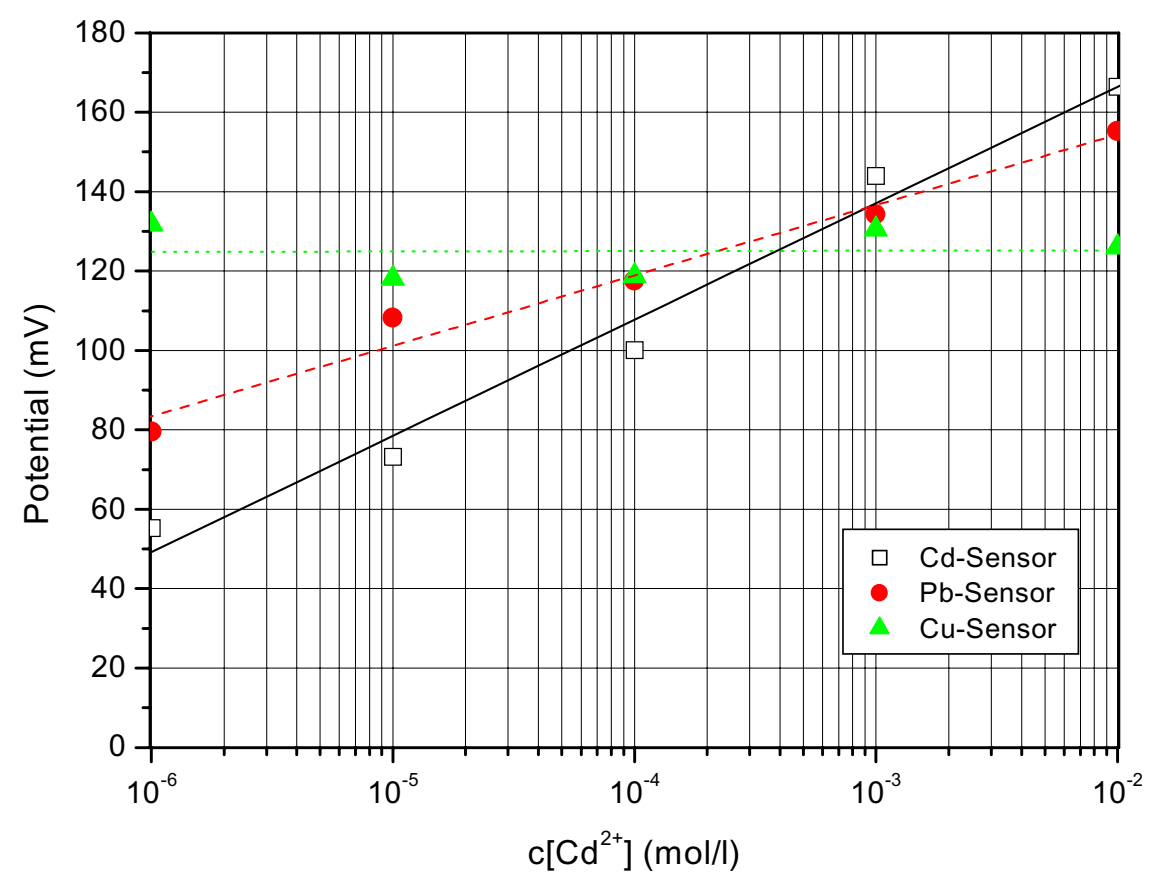

Figure 7. Calibration curves of the single sensors of the thin-film sensor array towards Cd2+ions.

With increasing ion concentrations from $10^{-6} \mathrm{~mol} / 1$ to $10^{-2} \mathrm{~mol} / \mathrm{l}$ and vice versa, the potentiometric response of the sensor array has been recorded. In the diagram, the sensitivity towards $\mathrm{Cd}^{2+}$ of the $\mathrm{Cd}$ sensitive layer was about $24 \mathrm{mV} / \mathrm{dec}$. In contrast, the sensor response of the Pb-selective sensor was about $14 \mathrm{mV} / \mathrm{dec}$. For the $\mathrm{Cu}$-selective sensor, a sensitivity to varying concentrations of $\mathrm{Cd}^{2+}$ of less than $1 \mathrm{mV} / \mathrm{dec}(0.2 \mathrm{mV} / \mathrm{dec})$ has been observed. As expected, the highest sensitivity was found for the Cd-type sensor, which detects its primary ion with highest sensitivity. Nonetheless, some crosssensitivity exists for the Pb-type sensor within the developed sensor array. This selectivity behaviour is also well-known from the macroscopic bulk electrodes and not originated from the thin-film sensor itself [8]. The Cu-type sensor posesses almost no cross-selectivity.

Further electrochemical measurements have been performed in $\mathrm{Pb}\left(\mathrm{NO}_{3}\right)_{2}$ - and $\mathrm{Cu}\left(\mathrm{NO}_{3}\right)_{2}$-solutions, too. The results obtained are comparable to the sensor behaviour of conventional bulk electrodes in terms of sensitivity and selectivity. For both, the $\mathrm{Pb}\left(\mathrm{NO}_{3}\right)_{2}$ - and $\mathrm{Cu}\left(\mathrm{NO}_{3}\right)_{2}$ - test samples, the Cd-type sensor shows the highest "interference" towards $\mathrm{Pb}^{2+}$-and $\mathrm{Cu}^{2+}$-ions, respectively. 


\section{Conclusions and Perspectives}

A first step towards a microfabricated thin-film sensor array on the basis of chalcogenide glass materials has been presented. The chalcogenide glass-based sensor array has been prepared by methods of silicon planar technology. As a new tool to deposit various chalcogenide glass materials ( $\mathrm{Pb}-\mathrm{Ag}$-As-I-S, Cd-Ag-As-I-S, Cu-Ag-As-Se) as thin films, the pulsed laser deposition technique emerged as an excellent method. It has been demostrated that it is possible to deposit three different chalcogenide glass thin films onto one silicon substrate by means of PLD process. The thickness of the thin films were about $500 \mathrm{~nm}$. The thin films have been physically characterized by means of SEM and RBS, and electrochemically characterized by means of ion-selective potentiometry. The chalcogenide glass-based thin-film sensor array exhibits a nearly Nernstian response to the respective primary ions such as $\mathrm{Pb}, \mathrm{Cu}$ and $\mathrm{Cd}$ in the concentration range of $10^{-6}-10^{-2} \mathrm{~mol} / \mathrm{l}$ for $\mathrm{Pb}^{2+}-$ and $\mathrm{Cd}^{2+}$-ions and $10^{-7}$ $10^{-3} \mathrm{~mol} / \mathrm{l}$ for $\mathrm{Cu}^{2+}$-ions. The results obtained in terms of cross-sensitivities, i.e. selectivities, are comparable to those measurements achieved with conventional bulk electrodes [8].

The presented paper demonstrates a good feasibility to design a compact thin-film sensor array based on chalcogenide glass materials. Future experiments will deal with the combination of several sensors of the thin-film array together with artificial neural networks (ANN) for data processing. Therewith, not only solutions that contain one ion but also complex mixtures could be analyzed. Moreover, the cross-sensitivity of the single sensors can be advantageously utilized to realize an "electronic tongue"-type thin-film sensor array. Basing on the presented results and with regard to practical applications, in the future, the authors plan to develop a "portable $\underline{e}$ lectrochemical testsystem for $\underline{r}$ eal-time $\underline{a}$ nalysis (PETRA)".

\section{Acknowledgements}

The authors gratefully thank Theodor Doll, Holger Ecken, Jürgen Müller, Angelika Pracht and Regina Stockmann for valuable discussions, and the Ministerium für Schule, Weiterbildung, Wissenschaft und Forschung des Landes Nordrhein-Westfalen for financial support.

\section{References}

1. Ahlers, H. Multisensorikpraxis, Springer-Verlag: Berlin, Heidelberg; New York, 1996, pp 182365.

2. Henze, G.; Köhler, M.; Lay, J.P. Umweltdiagnostik mit Mikrosystemen, Wiley-VCH: Weinheim, 1999, pp 1-62.

3. D'Amico, A.; Di Natale, C.; Paolesse, R. Portraits of gasses and liquids by arrays of nonspecific chemical sensors: trends and perspectives. Sens. Act. B 2000, 68, 324-330.

4. Mourzina, Yu. G.; Schubert, J.; Zander, W.; Legin, A.; Vlasov, Yu. G.; Lüth, H.; Schöning, M.J. Development of multisensor systems based on chalcogenide thin film chemical sensors for the simultaneous multicomponent analysis of metal ions in complex solutions. Electrochim. Act. 2001, $47,251-258$. 
5. Vlasov, Yu.G.; Legin, A.V. Non-selective chemical sensors in analytical chemistry: from "electronic nose" to "electronic tongue". Fresenius J. Anal. Chem. 1998, 361, 255-260.

6. Vlasov, Yu.; Legin, A.; Rudnitskaya, A. Electronic tongues and their analytical application. Anal. Bioanal. Chem. 2002, 373, 136-146.

7. Vlasov, Yu.G.; Bychkov, E.A. Ion-selective chalcogenide glass electrodes. Ion- selective Electrode Rev. 1987, 9, 5-93.

8. Vlasov, Yu.G.; Legin, A.; Rudnitskaya, A. Cross-sensitivity evaluation of chemical sensors for electronic tongue: determination of heavy metal ions. Sens. Act. B 1997, 44, 532-537.

9. Schöning, M.J.; Mourzina Yu.G.; Schubert J.; Zander W.; Legin, A.; Vlasov, Yu.G.; Lüth, H. Pulsed laser deposition - an innovative technique for preparing inorganic thin films. Electroanal. 2001, 13 (8-9), 727-732.

10. Schöning, M.J.; Mourzina, Yu.G.; Schubert, J.; Zander, W.; Legin, A.; Vlasov, Yu.G.; Lüth, H. Can pulsed laser deposition serve as an advanced technique in fabricating chemical sensors ? Sens. Act. B 2001, 78, 273-278.

11. Vlasov, Yu.G.; New solid-state ion-selective electrodes - sensors for chemical analysis of solutions. Fresenius Z. Anal. Chem. 1989, 355, 92-99.

12. Fassbender, F.; Schmitt, G.; Schöning, M.J.; Lüth H.; Buß, G.; Schultze, J.-W. Optimization of passivation layers for corrosion protection of silicon-based microelectrode arrays. Sens. Act. B 2000, 68, 128-133.

13. Schöning, M.J.; Schmidt, C.; Schubert, J.; Zander, W.; Mesters, S.; Kordos, P.; Lüth, H.; Legin, A.; Seleznev, B.; Vlasov, Yu.G. Thin film sensors on the basis of chalcogenide glass materials prepared by pulsed laser deposition technique. Sens. Act. B 2000, 68, 254-259.

14. Mourzina, Yu.G.; Schöning, M.J.; Schubert, J.; Zander, W.; Legin, A.; Vlasov, Yu.G.; Kordos, P.; Lüth, H. A new thin-film $\mathrm{Pb}$ microsensor based on chalcogenide glasses. Sens. Act. B 2000, 71, 13-18.

Sample Availability: Available from the authors.

(C) 2002 by MDPI (http://www.mdpi.net). Reproduction is permitted for noncommercial purposes. 